\title{
Gestión Municipal, midiendo la Incidencia del Presupuesto Participativo y el Desempeño Laboral en ella; caso: Municipio de La Molina, 2020
}

\section{Municipal Management, measuring the Incidence of the Participatory Budget and Labor Performance; case: Municipality of La Molina, 2020}

Daniel Ricardo Silva Siu, PhD (c)

https://orcid.org/0000-0003-1783-6261

Universidad César Vallejo, Perú

María Auxiliadora Guerrero Bejarano, DBA

https://orcid.org/0000-0002-1412-5870

Universidad Internacional del Ecuador, Ecuador

Ulises Córdova García, PhD

https://orcid.org/0000-0002-0931-7835

Universidad César Vallejo, Perú

Autor para correspondencia: dsilva@pobox.com; maguerrerobe@uide.edu.ec; ucordovag@ucvvirtual.edu.pe

Fecha de recepción: 29 de julio del 2020 - Fecha de aceptación: 15 de agosto del 2020

\section{Resumen}

El principal objetivo de este trabajo fue encontrar si el desempeño de los trabajadores y la forma de manejar el presupuesto afecta los resultados obtenidos por la administración municipal, utilizando como el caso de estudio a la Municipalidad de La Molina, en Lima Perú, estudio cuantitativo, se encuestó a trabajadores de la municipalidad en mención, cien en total, utilizando tres encuentas previamente validadas por expertos, todas las herramientas poseen cuestionarios con escalas de likert se midió la confibilidad con Alpha de Crombach. Se demostró que en este caso el presupuesto participativo y el desempeño laboral tienen incidencia en la gestión del municipio de La Molina.

Palabras claves: presupuesto participativo; desempeño laboral; gestión municipal

\begin{abstract}
The main objective of this work was to find out if the performance of the workers and the way of managing the budget affects the results obtained by the municipal administration, using as a case study the Municipality of La Molina, in Lima Peru, a quantitative study, workers of the mentioned municipality were surveyed, 100 in total, using three surveys previously validated by experts, all the tools have questionnaires with Likert scales and the reliability was measured with Alpha de
\end{abstract}


Cronbach. It was shown that in this case, participatory budgeting and job performance have an impact on the management of the municipality of La Molina.

Key words: participatory budgeting; job performance; municipal management

\section{Introducción}

El desarrollo de las ciudades es responsabilidad de sus ciudadanos tanto como de sus gobernantes, esto debido a que son estos primeros los que mediante el voto democrático eligen a los segundos para administrar los recursos públicos. En diferentes países ya se ha implementado de forma exitosa el presupuesto participativo como una forma en la que los ciudadanos se involucren en la gestión de las ciudades. En Latinoamérica se encuentra el caso de Porto Alegre en Brasil desde 1989, obteniendo como resultado mejoras en servicios básicos (Tarso y De Souza, 2015), en Europa, España en algunas ciudades entre las que se destacan Sevilla, Getafe, Córdova entre otras. De acuerdo con Martínez y Arenas (2013), en Argentina algunos municipios han tenido buenos resultados con la implementación de este tipo de presupuesto.

Perú tiene poco tiempo en la implementación del PP, se espera que las autoridades generen las condiciones para su desarrollo y que se tome en cuenta los ejemplos de los países antes mencionados (Francés, Carrillo y Sanchís, 2015:8). En el distrito de La Molina, ubicado en el sur de la ciudad de Lima, los ciudadanos resienten la falta de involucramiento que tienen en la gestión de su municipio; durante algunos años las distintas administraciones municipales han tenido bajo desempeño debido al incumplimiento de sus objetivos.

Estudios previos demuestran la evolución de ciudades que han aplicado el presupuesto participativo, en países como México, Chile y República Dominicana, dónde se encuentra que las leyes y la voluntad de los alcaldes pueden ser factores que permitan una mejor implementación del PP (Johnson 2017; García y Téllez 2018; Garrido y Montesinos 2018).

La teoría de la democracia participativa da vida al presupuesto participativo, en esta se establece la importancia de incluir a los ciudadanos en las decisiones de los municipios (Barragán, Romero y Sanz, 2015); desde la década de los 60 se fomenta la participación como resultado de los pobres resultados de los gobiernos locales junto con factores de los ideales socialistas (Macpherson 1977; Pateman 2014). Se sostiene que la participación de los ciudadanos en las decisiones de los gobiernos genera mayores nivels de ética, protege los intereses civiles, así como fomenta la educación y compromiso de los civiles en el desarrollo de las ciudades y de los países.

De acuerdo con Lawrence (2018) el desempeño laboral se define como la manera en la que los empleados realizan su trabajo, lo que tendría un impacto en los resultados obtendos por las organizaciones (Robbins 2004). El desempeño de acuerdo con Chiavenato (2004), puede ser medido lo que permite establecer las habilidades de los trabajadores y obtener los mejores resultados; permitiendo a los jefes o supervisores decidir sobre las formas en las que se distribuyen tareas para estimular mejores cumplimientos (Guillen, 2000), adicionalmente la medición de los resultados obtenidos permite conocer los aspectos que se pueden mejorar así como si existen factores que no se pueden controlar que afectan a los trabajadores y su comportamiento en el trabajo (Wherter y Davis, 2001). 
De acuerdo con la revisión de la literatura realizada, se generaron las siguientes preguntas de investigación: general ¿Cómo incide el PP y el DL en la gestión municipal de la municipalidad de La Molina, 2020?; especificas ¿Cómo incide el PP y el DL en el planeamiento y desarrollo municipal?; ¿Cómo incide el PP y el DL en la organización de la Municipalidad?; ¿Cómo incide el PP y el DL en los servicios municipales de la Municipalidad de La Molina, 2020?, de las que se desprendieron una hipótesis general y tres hipótesis específicas.

\section{Método}

Estudio cuantitativo de paradigma positivista, método hipotético deductivo (Bastar, 2012; Hernández, Fernández y Baptista, 2014; Zavala, 2007). Estudio descriptivo explicativo, causal y transversal que busca demostrar la relación entre las variables, dos independientes: presupuesto participativo y desempeño laboral y una independiente: gestión municipal.

Muestra probabilística de conveniencia, se trabajó con los empleados del Municipio de La Molina, como criterio de restricción y debido a las características de la población se definió que los participantes tuvieran al menos 6 meses trabajando. Participaron cien empleados 38 pertenecían al área de presupuesto, 35 a la gerencia y 27 a desarrollo urbano.

Los cuestionarios utilizados fueron validados por cinco expertos y su confiabilidad medida a través del Alfa de Cronbach obteniendo los siguientes resultados: PP 0.937; DL 0.934 y GM 0.887 se concluye que existe alta confiabilidad de los instrumentos. Todos los encuestados participaron de manera voluntaria e informada, los datos se analizaron con SPSS, se utilizó la regresión logística ordinal para el análisis de las variables.

\section{Resultados}

Esta investigación analizó los datos utilizando la regresión ordinaria lineal que permite analizar los datos inferencialmente en casos como este; en el caso de la hipótesis general:

Ho: El presupuesto participativo y el desempeño laboral no inciden significativamente en la gestión municipal de la Municipalidad de la Molina, 2020.

Ha: El presupuesto participativo y el desempeño laboral inciden significativamente en la gestión municipal de la Municipalidad de la Molina, 2020.

\section{Y: Gestión municipal; $\mathrm{X}_{1}$ : Presupuesto participativo; $\mathrm{X}_{2}$ : Desempeño laboral}

Los resultados obtenidos se muestran en las tablas 1 y2, en la primera se confirma la bondad de ajuste necesaria para utilizar el modelo de regresión lineal ordinaria en este estudio 
Tabla 1

Información de ajuste de modelo para Hipótesis general

\begin{tabular}{llllc}
\hline Modelo & Logaritmo de la verosimilitud-2 & Chi cuadrado & gl & Sig. \\
\hline Solo intercepción & 52,438 & & & \\
Final & 32,921 & 19,517 & 4 &, 001 \\
\hline
\end{tabular}

Función de enlace: Logit.

Tabla 2

Regresión logística Ordinal de Hipótesis General

\begin{tabular}{|c|c|c|c|c|c|c|c|c|}
\hline \multicolumn{9}{|c|}{ Estimaciones de parámetro } \\
\hline & & & Error & & & & $95 \%$ de interval & o de confianza \\
\hline & & Estimación & estándar & Wald & $\mathrm{gl}$ & Sig. & Límite inferior & Límite superior \\
\hline \multirow[t]{2}{*}{ Umbral } & {$[\mathrm{cc}=1.00]$} & $-6,010$ & 1,059 & 32,188 & 1 & 0,000 & $-8,086$ & $-3,934$ \\
\hline & {$[\mathrm{cc}=2.00]$} & $-1,289$ & ,839 & 2,361 & 1 & 0,124 & $-2,932$ &, 355 \\
\hline \multirow[t]{6}{*}{ Ubicación } & {$[a=1.00]$} & $-1,759$ & 1,109 & 2,516 & 1 & 0,113 & $-3,933$ & ,415 \\
\hline & {$[a=2.00]$} & $-1,489$ & 900 & 2,737 & 1 & 0,098 & $-3,254$ & 275 \\
\hline & {$[a=3.00]$} & $0^{\mathrm{a}}$ & . & . & 0 & . & . & . \\
\hline & {$[\mathrm{b}=1.00]$} & $-2,394$ & 1,104 & 4,706 & 1 & 0,030 & $-4,558$ &,- 231 \\
\hline & {$[b=2.00]$} & $-1,196$ & ,509 & 5,522 & 1 & 0,019 & $-2,194$ &,- 198 \\
\hline & {$[b=3.00]$} & $0^{\mathrm{a}}$ & . & . & 0 & . & . & . \\
\hline
\end{tabular}

Función de enlace: Logit

$\underline{\text { a. Este parámetro está establecido en cero porque es redundante. }}$

En la tabla anterior se pudo observar el nivel 'No óptimo' (1) de la variable 'Gestión Municipal' presenta valor ' $p$ ' significativo $\left(\mathrm{p}_{(1)}=0.000<0.05\right)$ con coeficiente de Wald superior a 4.00 (De La Garza et al. 2013) lo que indica, un nivel influenciado por las variables independientes del estudio. No existe influencia de la variable 'Gestión Municipal' en las variables independientes. Precisando la condición de influencia de las variables independientes $\left(\mathrm{X}_{1} \mathrm{y}_{2}\right)$ sobre la dependiente (Y), se indica dos niveles de la variable independiente 'Desempeño laboral' significativos; siendo el nivel 1 (No óptimo) con un valor $\mathrm{p}=0.000<0.05 \mathrm{y}$ el nivel 2 (Básico) con un valor $\mathrm{p}=0.019<$ 0.05. Se rechazar la hipótesis nula, precisando que el presupuesto participativo y el desempeño laboral inciden significativamente en la gestión de la Municipalidad de la Molina, 2020.

\section{Prueba de hipótesis específica 1}

Ha: El presupuesto participativo y el desempeño laboral incide significativamente en el planeamiento y desarrollo municipal de la Municipalidad de la Molina, 2020.

Ho: El presupuesto participativo y el desempeño laboral no incide significativamente en el planeamiento y desarrollo municipal de la Municipalidad de la Molina, 2020.

Y: Planeamiento y desarrollo municipal, $\mathrm{X}_{1}$ : Presupuesto participativo y $\mathrm{X}_{2}$ : desempeño laboral 
Tabla 3

Información de ajuste de modelo para Hipótesis especifica 1

\begin{tabular}{|c|c|c|c|c|}
\hline Modelo & $\begin{array}{l}\text { Logaritmo de la } \\
\text { verosimilitud-2 }\end{array}$ & Chi cuadrado & gl & Sig. \\
\hline Solo intercepción & 525,220 & & & \\
\hline Final & 22,283 & 32,936 & 4 &, 000 \\
\hline
\end{tabular}

Función de enlace: Logit

Se presenta la información de ajuste de modelo en la tabla 3, donde el ratio de probabilidad de Chi cuadrado fue de 32,936 con un valor de significancia de ,000 < a ,05 que indica que el modelo se ajusta a estudio de la RLO.

\section{Tabla 4}

Calidad de ajuste de modelo

\begin{tabular}{lr}
\hline \multicolumn{2}{l}{ Pseudo R cuadrado } \\
\hline Cox y Snell &, 281 \\
Nagelkerke &, 378 \\
McFadden &, 242 \\
\hline Función de enlace: Logit.
\end{tabular}

Se usaron los coeficientes: Pseudo R-cuadrado de Cox y Snell, Nagelkerke y McFadden para medir la calidad de ajuste del modelo. Los resultados indicaron una buena calidad de ajuste para el análisis estadístico propuesto.

\section{Tabla 5}

Regresión logística Ordinal para Hipótesis especifica 1

\begin{tabular}{|c|c|c|c|c|c|c|c|c|}
\hline \multicolumn{9}{|c|}{ Estimaciones de parámetro } \\
\hline & & \multirow[b]{2}{*}{ Estimación } & \multirow[b]{2}{*}{ Error estándar } & \multirow[b]{2}{*}{ Wald } & \multirow[b]{2}{*}{ gl } & \multirow[b]{2}{*}{ Sig. } & \multicolumn{2}{|c|}{$95 \%$ de intervalo de confianza } \\
\hline & & & & & & & Límite inferior & Límite superior \\
\hline \multirow[t]{2}{*}{ Umbral } & {$[a 3=1]$} & $-6,600$ & 1,148 & 33,040 & 1 & ,000 & $-8,850$ & $-4,349$ \\
\hline & {$[\mathrm{a} 3=2]$} &,- 785 &, 776 & 1,022 & 1 & 312 & $-2,306$ & ,737 \\
\hline \multirow[t]{6}{*}{ Ubicación } & {$[a=1.00]$} & $-2,282$ & 1,179 & 3,750 & 1 & 053 & $-4,593$ &, 028 \\
\hline & {$[a=2.00]$} & $-2,309$ & ,906 & 6,502 & 1 & 011 & $-4,084$ &,- 534 \\
\hline & {$[a=3.00]$} & $0^{\mathrm{a}}$ & . & . & 0 & . & . & . \\
\hline & {$[\mathrm{b}=1.00]$} & $-3,989$ & 1,257 & 10,069 & 1 & ,002 & $-6,452$ & $-1,525$ \\
\hline & {$[b=2.00]$} & $-2,128$ & ,838 & 6,447 & 1 & ,011 & $-3,770$ &,- 485 \\
\hline & {$[b=3.00]$} & $0^{\mathrm{a}}$ &. & . & 0 & . & . & . \\
\hline
\end{tabular}

Función de enlace: Logit

a. Este parámetro está establecido en cero porque es redundante.

Se observa que el nivel 'No óptimo' (1) de la variable dependiente 'Planeamiento y desarrollo municipal' presenta valor ' $p$ ' significativo $\left(\mathrm{p}_{(1)}=0.000<0.05\right)$ teniendo coeficiente de Wald superior a 4.00 (De La Garza et al. 2013) lo que indica un nivel influenciado por las variables independientes. No se registró influencia a los niveles 2 (Básico) y 3 (Óptimo) de la variable 'Planeamiento y desarrollo municipal' por parte de las variables independientes. 
Precisando la condición de influencia de las variables independientes sobre la dependiente, se indica que un nivel de la variable independiente fue significativo; siendo el nivel 2 (Básico) con un valor $\mathrm{p}=0.011<0.05$ y dos niveles de la variable Desempeño laboral, siendo el nivel 1 (No óptimo) con un valor $\mathrm{p}=0.002<0.05 \mathrm{y}$ el nivel 2 (Básico) con un valor $\mathrm{p}=0.011<0.05$. Se rechaza la hipótesis nula, el presupuesto participativo y el desempeño laboral incide significativamente en el planeamiento y desarrollo municipal de la Molina, 2020.

\section{Prueba de hipótesis específica 2}

Ha: El presupuesto participativo y el desempeño laboral incide significativamente en la organización de la Municipalidad de la Molina, 2020.

Ho: El presupuesto participativo y el desempeño laboral no incide significativamente en la organización de la Municipalidad de la Molina, 2020.

Y: Organización; $\mathrm{X}_{1}$ : Presupuesto participativo y $\mathrm{X}_{2}$ : Desempeño laboral

\section{Tabla 6}

Información de ajuste de modelo

\begin{tabular}{lllll}
\hline Modelo & $\begin{array}{l}\text { Logaritmo de la } \\
\text { verosimilitud-2 }\end{array}$ & Chi cuadrado & gl & Sig. \\
\hline $\begin{array}{l}\text { Solo intercepción } \\
\text { Final }\end{array}$ & $\begin{array}{c}56,957 \\
25,906\end{array}$ & 31,050 & 4 &, 000 \\
\hline Función de enlacer Logit & & & 4
\end{tabular}

Función de enlace: Logit

En la tabla 6, se encuentra la información de ajuste, donde el Chi cuadrado fue de 31,050 con un valor de significancia de, $000<$ a ,05 que indica que el modelo se ajusta a estudio de la RLO.

\section{Tabla 7}

Calidad de ajuste de modelo

\begin{tabular}{ll}
\hline \multicolumn{2}{l}{ Pseudo R cuadrado } \\
\hline Cox y Snell &, 267 \\
Nagelkerke &, 343 \\
McFadden &, 206 \\
\hline \multicolumn{2}{l}{ Función de enlace: Logit. }
\end{tabular}

En la tabla 7 se muestran los valores de los coeficientes utilizados que demuestran que existe buen ajuste para utilizar RLO en la comprobación de esta hipótesis. 


\section{Tabla 8}

RLO para Hipótesis especifica 2

\section{Estimaciones de parámetro}

\begin{tabular}{|c|c|c|c|c|c|c|c|c|}
\hline & \multirow[b]{2}{*}{ Estimación } & \multirow[b]{2}{*}{ Error estándar } & \multirow[b]{2}{*}{ Wald } & \multirow[b]{2}{*}{ gl } & \multirow[b]{2}{*}{ Sig. } & \multicolumn{2}{|c|}{$95 \%$ de intervalo de confianza } \\
\hline & & & & & & & Límite inferior & Límite superior \\
\hline \multirow[t]{2}{*}{ Umbral } & {$[\mathrm{b} 3=1]$} & $-5,157$ & ,954 & 29,208 & 1 &, 000 & $-7,027$ & $-3,287$ \\
\hline & {$[\mathrm{b} 3=2]$} &,- 176 &, 721 &, 059 & 1 & ,808 & $-1,589$ & 1,238 \\
\hline \multirow[t]{6}{*}{ Ubicación } & {$[a=1.00]$} & $-2,283$ & 1,104 & 4,277 & 1 & 039 & $-4,446$ &,- 119 \\
\hline & {$[a=2.00]$} & $-1,566$ &, 845 & 3,440 & 1 & ,064 & $-3,222$ & ,089 \\
\hline & {$[a=3.00]$} & $0^{\mathrm{a}}$ & . & . & 0 & . & . & . \\
\hline & {$[b=1.00]$} & $-3,281$ & 1,062 & 9,548 & 1 &, 002 & $-5,362$ & $-1,200$ \\
\hline & {$[b=2.00]$} & $-1,369$ & ,662 & 4,282 & 1 & 039 & $-2,667$ &,- 072 \\
\hline & {$[b=3.00]$} & $0^{\mathrm{a}}$ & . & . & 0 & . & . & . \\
\hline
\end{tabular}

Función de enlace: Logit

a. Este parámetro está establecido en cero porque es redundante.

Se observa que el nivel 'No óptimo' (1) de la variable dependiente; presenta valor 'p' significativo $\left(\mathrm{p}_{(1)}=0.000<0.05\right)$ teniendo coeficiente de Wald superior a 4.00 (De La Garza $e t$ al. 2013) lo que indica que es existe influencia de las variables independiente. No se registra influencia para los niveles 2 (Básico) y 3 (Óptimo) de la variable 'Organización' por parte de las variables independientes. Precisando la condición de influencia de las variables independientes $\left(\mathrm{X}_{1}\right.$ y $\left.\mathrm{X}_{2}\right)$ sobre la dependiente $(\mathrm{Y})$, se indica que un nivel de la variable independiente 'Presupuesto participativo' $\left(\mathrm{X}_{1}\right)$ fue significativo; siendo el nivel 1 (No óptimo) con un valor $\mathrm{p}=$ $0.039<0.05$ y dos niveles de la variable independiente 'Desempeño laboral' $\left(\mathrm{X}_{2}\right)$, siendo el nivel 1 (No óptimo) con un valor $\mathrm{p}=0.002<0.05 \mathrm{y}$ el nivel 2 (Básico) con un valor $\mathrm{p}=0.039<0.05$. Se rechaza la hipótesis nula, afirmando que el presupuesto participativo y el desempeño laboral si incide significativamente en la organización de la Municipalidad de la Molina, 2020.

\section{Prueba de hipótesis específica 3}

Ha: El presupuesto participativo y el desempeño laboral incide significativamente en los servicios municipales de la Municipalidad de la Molina, 2020.

Ho: El presupuesto participativo y el desempeño laboral no incide significativamente en los servicios municipales de la Municipalidad de la Molina, 2020.

Y: Servicios municipales; $\mathrm{X}_{1}$ : Presupuesto participativo y $\mathrm{X}_{2}$ : Desempeño laboral

\section{Tabla 9}

Información de ajuste de modelo

\begin{tabular}{lllll}
\hline Modelo & $\begin{array}{l}\text { Logaritmo de la } \\
\text { verosimilitud-2 }\end{array}$ & Chi cuadrado & gl & Sig. \\
\hline $\begin{array}{l}\text { Solo intercepción } \\
\text { Final }\end{array}$ & 56,162 & & & \\
\hline
\end{tabular}

Función de enlace: Logit. 
En la tabla 9, se presentó la información de ajuste de modelo, donde le ratio de probabilidad de Chi cuadrado fue de 26,298 con un valor de significancia de ,000 < a ,05 que indica que el modelo se ajusta a estudio de la RLO.

\section{Tabla 10}

\section{Calidad de ajuste de modelo}

\begin{tabular}{ll}
\hline Pseudo R cuadrado & \\
\hline Cox y Snell &, 261 \\
Nagelkerke &, 271 \\
McFadden &, 137 \\
\hline
\end{tabular}

Función de enlace: Logit.

En la tabla 10 se muestra los resultados de los coeficientes de Pseudo R-cuadrado de Cox y Snell, Nagelkerke y McFadden. Sus cálculos con Cox y Snell, midió $(, 261)$ que casi no alcanzan el valor de 1 , pero se obtuvo un rango mayor a 0,1 por lo que se obtiene un buen ajuste. Con respecto a Mc Fadden tiene un rango de $0 \leq \mathrm{R} 2 \leq 1$, pero raramente se aproxima a 1 con lo que se considera buena calidad de ajuste cuando están en $0,2 \leq \mathrm{R} 2 \leq 0,4$; se obtuvo $(, 137)$. Por ello se utiliza como medida de bondad de ajuste Nagelkerke $(, 271)$ dentro de los parámetros que indica De la Garza, Morales y Gonzales (2013) mayor a $(, 150)$ para calidad de la prueba.

\section{Tabla 11}

Regresión logística Ordinal de Hipótesis específica 4

\begin{tabular}{|c|c|c|c|c|c|c|c|c|}
\hline \multicolumn{9}{|c|}{ Estimaciones de parámetro } \\
\hline & & \multirow[b]{2}{*}{ Estimación } & \multirow[b]{2}{*}{ Error estándar } & \multirow[b]{2}{*}{ Wald } & \multirow[b]{2}{*}{$\mathrm{gl}$} & \multirow[b]{2}{*}{ Sig. } & \multicolumn{2}{|c|}{$95 \%$ de intervalo de confianza } \\
\hline & & & & & & & Límite inferior & Límite superior \\
\hline \multirow[t]{2}{*}{ Umbral } & {$[\mathrm{d} 3=1]$} & $-3,724$ & ,824 & 20,442 & 1 &, 000 & $-5,339$ & $-2,110$ \\
\hline & {$[\mathrm{d} 3=2]$} &,- 500 & ,724 &, 477 & 1 & 490 & $-1,919$ & ,919 \\
\hline \multirow[t]{6}{*}{ Ubicación } & {$[a=1.00]$} & $-1,742$ & 990 & 3,096 & 1 & 078 & $-3,683$ & ,198 \\
\hline & {$[a=2.00]$} & $-1,760$ & ,818 & 4,625 & 1 & 032 & $-3,364$ &,- 156 \\
\hline & {$[a=3.00]$} & $0^{\mathrm{a}}$ & . & . & 0 & . & . & . \\
\hline & {$[b=1.00]$} & $-3,096$ & ,968 & 10,223 & 1 &, 001 & $-4,995$ & $-1,198$ \\
\hline & {$[b=2.00]$} & $-1,012$ &, 508 & 3,970 & 1 &, 046 & $-2,007$ &,- 017 \\
\hline & {$[b=3.00]$} & $0^{\mathrm{a}}$ & . & . & 0 & . & . & . \\
\hline
\end{tabular}

Función de enlace: Logit

a. Este parámetro está establecido en cero porque es redundante.

Se observa que el nivel 'No óptimo' (1) de la variable dependiente que presenta valor 'p' significativo $\left(\mathrm{p}_{(1)}=0.000<0.05\right)$ teniendo coeficiente de Wald superior a 4.00 (De La Garza et al. 2013) lo que indica un nivel influenciado por las variables independientes. No se registra influencia para los niveles 2 (Básico) y 3 (Óptimo) de la variable 'Servicios municipales' por parte de las variables independientes. Precisando la condición de influencia de las variables independientes $\left(\mathrm{X}_{1}\right.$ y $\left.\mathrm{X}_{2}\right)$ sobre la dependiente $(\mathrm{Y})$, se indica que un nivel de la variable independiente 'Presupuesto participativo' $\left(\mathrm{X}_{1}\right)$ fue significativo; siendo el nivel 2 (Básico) con un valor $\mathrm{p}=0.032<0.05 \mathrm{y}$ de la variable independiente 'Desempeño laboral' $\left(\mathrm{X}_{2}\right)$ fue significativo el nivel 1 (No óptimo) con un valor $\mathrm{p}=0.001<0.05 \mathrm{y}$ el nivel 2 (Básico) con un valor $\mathrm{p}=0.046<$ 
0.05. Se rechaza la hipótesis nula, afirmando que el presupuesto participativo y el desempeño laboral incide significativamente en los servicios de la Municipalidad de la Molina, 2020.

\section{Conclusiones y recomendaciones}

Se concluye que el presupuesto participativo y el desempeño laboral tienen influencia sobre la gestión del Municipio de la Molina, lo que implica una oportunidad para los gobiernos locales de mejorar sus resultados a partir del involucramiento de sus ciudadanos en la planificación y toma de decisiones del posible manejo de recursos con los que se espera generar mejores rendimientos (Barragán et all, 2015).

En los diferentes análisis realizados en este estudio se demostró que para el caso del Municipio de La Molina, las variables independientes influyen en las distintas dimensiones o factores que se desprenden de la gestión municipal, lo que significa que tanto el involucramiento de los ciudadanos como la motivación que los empleados municipales tienen al momento de desarrollar sus tareas, influyen en los resultados de organización, servicios y desarrollo de las actividades municipales (Frances et all, 2015).

Diferentes municipios a lo largo del mundo utilizan la administración participativa para permitir a los ciudadanos involucrarse de forma activa en las decisiones de los gobiernos locales, por lo que se recomienda a las autoridades de los municipios del Perú, seguir los ejemplos antes citados para mejorar el bienestar de sus localidades; estos cambios podrán permitirles obtener mejores resultados en el cumplimiento de los objetivos planteados por cada administración municipal.

Se recomienda continuar estos estudios extendiendo la población a distintos municipios con el fin de conseguir análisis comparativos que permitan comprender mejor la importancia del presupuesto participativo para el desarrollo de los pueblos.

\section{Bibliografía}

Barragán, V., Sanz, J. M. y Romero, R. (2015) Indicadores para el análisis de las propuestas ciudadanas en presupuestos participativos. Hacia el derecho a la ciudad. Chasqui Revista Latinoamericana de Comunicación, (129), 363-380. [Fecha de consulta 26 de junio de 2020]. ISSN: 1390-1079. Disponible en: https://www.redalyc.org/articulo.oa?id=160/16057397022

Bastar, S. (2012). Metodología de la Investigación. México, Red Tercer Milenio.

Chiavenato, I. (2004). Comportamiento Organizacional. México: Editorial Mc. Graw Hill,

De La Garza, J., Morales, B. N. y. Gonzales, B. A. (2013). Análisis estadístico multivariante. México: McGraw Hill.

Francés, F., Carrillo, A. y Sanchís, M. (2015). Presupuestos participativos. Guía metodológica para la implementación de un presupuesto participativo. España, Preparación Ediciones.

García M. y Téllez, L. (2018). El presupuesto participativo: un balance de su estudio y evolución en México. Perfiles Latinoamericanos, 26 (52), DOI:10.18504/pl2652-012-2018 
Garrido, F y Montesinos, E. (2018). El Presupuesto Participativo en Chile y República Dominicana: ¿Es determinante una Ley para el fortalecimiento de la democracia participativa? Rev. Uruguaya de Ciencia y. Política, 27 (2), 99-120. http://dx.doi.org/10.26851/rucp.28.3

Guillén, R.G. (2000) Psicología del trabajo para las relaciones laborales. 8th ed. España: S.A.U; McGraw Hill/Interamericana de España

Hernández, R., Fernández, R. y Baptista, P. (2014). Metodología de la Investigación. (6 ${ }^{\text {a }}$. Edición) México: Mc Graw Hill Education.

Johnson, C. (2017). Engaging Democracy: An Institutional Theory of Participatory Budgeting. (Doctoral dissertation, University of Washington) Recuperado de: https://url2.cl/dQWjk

Lawrence, A. (2018). The Top 5 Qualities That Lead to High Job Performance. PSI Select International. Recuperado de: selectinternational.com

Macpherson, C. B. (1977) La democracia liberal y su época. Madrid. Alianza editorial.

Martínez, C. y Arena, E. (2013). Experiencias y buenas prácticas en Presupuesto Participativo. UNICEF - Secretaría de Relaciones Parlamentarias de la Nación - Universidad Nacional de General Sarmiento. Recuperado de: https://www.unicef.org/evaldatabase/index_72958.html

Pateman, C. (2014) Participación y teoría democrática. 2da. Edición. Buenos Aires Argentina. Prometeo Libros. ISBN: 9789875746695

Robbins, S. (2004). Comportamiento Organizacional. (7ma. Edición) México: Prentice Hall.

Tarso, G y De Souza, U. (1999). Presupuesto participativo: la experiencia de Porto Alegre. Montevideo: Trilce, [ISBN 9974322049

Werther, WB, Davis K. (2001) Administración de Personal y de Recursos Humanos. 5th ed. México: McGraw Hill.

Zavala, F. (2007). Filosofía del México. México: Editorial Porrua, Lomas verde. 